\title{
On the Derivation of the Double Object Construction in Mandarin Chinese
}

\author{
Haojie Li \\ Faculty of English Language and Culture, Guangdong University of Foreign Studies, Guangzhou, China; \\ School of Foreign Languages, Southwest University of Political Science and Law, Chongqing, China \\ Zhigang Ma \\ Center of Linguistics and Applied Linguistics, Guangdong University of Foreign Studies, Guangzhou, China
}

\begin{abstract}
This paper adopts a Labeling approach to the analysis of the double object construction (DOC). In conformity with Chomsky's (2013, 2014, 2015) Labeling Algorithm (LA), a unified account is proposed of the derivation of DOC in Mandarin Chinese. It is argued that the pair-Merged element in a complex verb in the DOC is invisible for labeling and the normal labeling procedure for the Head will apply.
\end{abstract}

Index Terms - DOC, labeling algorithm, minimal search, simplest merge

\section{INTRODUCTION}

The following examples (1a) and (2a) are Double Object Constructions (DOC) and examples (1b) and (2b) are Dative Constructions (DC) from Mandarin Chinese and English. These two constructions are collectively referred to ditransitive constructions. The DOC is the focus of this paper.

(1) a. Zhangsan song Lisi yiben shu.

Zhangsan give Lisi one-CL book

'Zhangsan gave Lisi a book.'

b. Zhangsan song yiben shu gei Lisi.

Zhangsan give one-CL book to Lisi

'Zhangsan gave a book to LIsi.'

(2) a. John gave Mary a book.

b. John gave a book to Mary.

The DOC has always been one of the issues hotly debated in linguistic theory. There are two major problems in studying the DOC from a syntactic perspective. One is the Cases of the direct object (DO) and the indirect object (IO). The other is the relationship of the DOC and the dative construction. Some researchers maintain that there may be some transformational relationship between the DOC and the dative construction. So far, no consensus has been reached whether the DOC is base-generated or is transformed by movement via the dative construction.

The contents of this paper are arranged as follows: section two summarizes the main issues of the study of the DOC, the different analysis methods and the problems of them. The third section introduces the related content of Chomsky's (2013, 2014, 2015) Labeling Algorithm (LA). The fourth section analyzes the DOC in terms of the spirit of Chomsky's LA. The last section serves as a conclusion.

\section{RELATED RESEARCHES}

The study of the Double Object Construction (DOC) has always been one of the major topics in the literature of generative grammar, such as Chomsky (1975), Kayne (1984), Baker(1988), Aoun\&Li (1989), Barss\&Lasnik (1986), Larson (1988, 1990), Jackendoff (1990), Bowers (1993), Marantz (1993), Pesetsky (1995), Fujita (1996), Collins \&Thrainsson (1996), Radford (1997), Pylkkänen (2002), Anagnostopoulou (2003), Harley(2003), Beck\&Johnson (2004), Miyagawa\&Tsujioka (2004), Soh (2005), etc. have discussed the DOC. The main focuses of those discussions are the generation of the DOC and the problems of Cases of the two objects in the DOC.

The above-mentioned researches on the DOC can be divided into three categories: the transformational approach, the causation-possession approach and the applicative approach. We will tease apart the three categories in detail in the following sections.

\section{A. The Transformational Approach}

How is the DOC generated, is it base-generated or is it transformed from the DC? It is the focus of the transformational Approach which prescribes that the DOC and the DC are transformationally related. According to the analysis by Chomsky (1975), the DOC is transformed from the DC. Chomsky (1975) holds that (3c) is transformed from (3b) (DOC) and (3b) (DC) is transformed from (3a). 
(3) a. John [vp a letter [v, sent to Mary]]

b. John sent [vp a letter [v,t to Mary]]

c. John sent Mary a letter.

In (3a), the verb 'sent' and the complement 'to Mary' constitute one constituent, which does not contain DO 'a letter'. And then, the verb 'sent' raises to form DC. The generation of the DOC is through dative shift, viz., the IO is moved to the front of the DO.

Barss\&Lasnik (1986) finds six kinds of asymmetrical relations between IOs and Dos in DOCs.

(4) I showed John himself. (anaphor binding)

*I showed himself John.

(5) I denied each worker ${ }_{\mathrm{i}}$ his $_{\mathrm{i}}$ paycheck. (quantifier binding)

*I denied its $\mathrm{s}_{\mathrm{i}}$ owner each paycheck $\mathrm{k}_{\mathrm{i}}$.

(6) Which worker ${ }_{i}$ did you deny his $s_{i}$ paycheck? (weak crossover)

*Which paycheck $\mathrm{k}_{\mathrm{i}}$ did you deny $\mathrm{its}_{\mathrm{i}}$ owner?

(7) Who did you give which book? (superiority)

*Which book did you give who?

(8) I gave each man the other's watch. (each...the other)

*1 gave the other's trainer each lion.

(9) I gave no one anything. (negative polarity items)

*I gave anyone nothing.

These six asymmetries between IOs and DOs all involve the structural relationship of c-command, and IOs must c-command DOs. The observations of Barss \& Lasnik (1986) have a great influence on the later study of DOC.

Larson $(1988,1990)$ based on Chomsky's (1975) analysis of the DOC, proposed the concept of VP-shell in order to explain the asymmetry pointed out by Barss \& Lasnik (1986). Larson believes that the DC is base-generated, and the DOC is transformed from the DC through passivization.

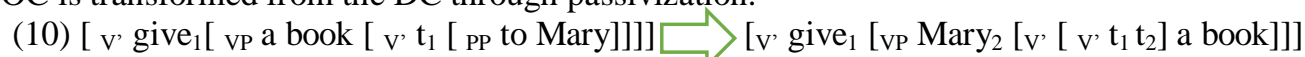

Aoun\&Li (1989) puts forward that the DOC is base-generated, and the DC is transformed from the DOC via passivization and right adjunction.

(11) [vp give [sc Mary [vp e a book]]] $\longrightarrow$ [vp give [ sc a book ${ }_{2}\left[\mathrm{vp}_{\mathrm{vP}}\right.$ e t $\mathrm{v}_{2}$ ] to Mary]]]

The transformation analysis seems to solve the problem of the c-commanding relationship between the DO and the IO and explains the reason why the two sentences have similar meanings. However, the differences in verb selection and semantic expression between the DOC and the DC do not support the transformation analysis (He 2003, 2009).

\section{B. The Causation-possession Approach}

In view of the problems of the "transformation analysis", some researchers have adopted the causation-possession analysis, and believe that the DOC and the DC have their own internal structures respectively. Researches using this analysis mostly use the semantics expressed by the DOC as the basis for the syntactic derivation. The semantic approach which holds that the basic meaning expressed by DOC is possession (Pesetsky1995, Harley2003 and Beck\&Johnson2004).

Green (1974: 156-167), after analyzing the semantic differences between the two constructions, points out that there is a special semantic relationship, that is, ownership (or possession) relationship between IOs and DOs (or possession) in the DOC.

In order to express this semantic relationship, Kayne (1984: 134) once proposes that the verb selects a small clause (SC) in the DOC. This SC indicates that IOs have DOs. However, in this construction, the IO and the DO are sister relations, which does not reflect the asymmetric c-command relationship between the two objects in the DOC.

Pesetsky (1995) absorbs the concept of hierarchical structures in Larson's (1988) analysis to reflect the asymmetries of the syntactic structures. In these two constructions proposed by Pesetsky, the verb chooses different prepositional phrases (PPs) as complements. In the DOC, the head of the PP is an empty preposition 'G' without phonetic representation, which represents the semantic relationship between the two objects, that is, the IO owns the DO, the IO is the specifier of G, and the DO is the complement of G. In the DC, the head of the PP is a preposition 'to' with a phonetic expression, the DO is its signifier, and the IO is its complement.

Harley (2003) proposes the Lexical Decomposition Analysis on the basis of Pesetsky (1995). The ditransitive verbs are divided into CAUSE+HAVE and CAUSE+ GO TO, which appear in the DOC and the DC respectively. The DOC means "the subject makes the IO have the DO". The DC means "the subject moves the DO to the IO".

Beck\&Johnson (2004) synthesizes the analyses of Kayne's (1984) SC analysis and Harley's (2003) Lexical Decomposition Analysis. They believe that the DOC and the DC have different internal structures, and there is no transformational relationship between the two constructions (Beck and Johnson 2004: 98). They use the adverb "again" as a test method to implement the in-depth semantic analysis of these two constructions. The conclusion is that there is a $\mathrm{SC}$ in the DOC, and the head of the SC is HAVE, which proves that there is no transformational relationship between the DOC and the DC.

\section{The Applicative Approach}


The applicative approach which argues that APPL construction and DOC have the same underlying structure (Pylkkänen2002, Anagnostopoulou 2003, Miyagawa\&Tsujioka 2004, and Soh 2005). It is argued that DOC and the dative construction are not derivationally related, and that possession meaning may not be representative of the DOC.

The use of Applicative morphemes usher IOs into the applicative construction, i.e., APPL construction in Bantu. The added argument is called the APPL argument. This APPL construction is similar to the English DOC. The difference is that in the APPL construction, the verbs have more complex morphological representations. To add the Applicative affix to the verb stem, for example (12) (Baker1988: 229; Bowers 2011:1198). The example is Chichewa in Bantu.

(12) a. Mbidzi zi-na-perek-a msampha kwa nkhandwe

Zebras SP-PASP-hand-ASP trap to fox

'The zebras handed the trap to the fox'

b. Mbidzi zi-na-perek-er-a nkhandwe msampha

Zebras SP-PASP-hand-APPL-ASP fox trap

'The zebras handed the fox the trap'

(12a) is the DC and (12b) is the APPL construction in Chichewa. There is the applicative affix 'er' attached to the verb. Baker (1988) and Marantz (1993) believe that the DOC and the APPL construction should have the same internal structure, the DOC should also have an applicative morpheme. The difference between the DOC and the APPL construction is that the former's applicative morpheme is covert, while the latter's applicative morpheme is overt.

Marantz (1993) proposes an analysis of the DOC based on the fact of Bantu corpus. He maintains that there is an applicative head in the DOC, which leads the IO.

(13) a. [VP DP [V' V [APPLP DP [APPL' APPL [VP [V DP]]]]]]

b. [VP DP [V'V [VP V [APPLP DP [APPL' [ APPL DP]]]]]]

The DOC proposed by Marantz is similar to the following example (13a). In this construction, the APPL head selects the VP, which is composed of the double transitive verb and the DO, as the complement. This head connects the event expressed by the VP and the IO in its specifier position. Marantz holds that the DOC has the causative semantic features due to this head, which also makes the IO own the role of "owner" in the DOC. In this construction, both the causative and possessive semantics are realized, and the structural relationship between the two objects is also reflected. Marantz believes that there is no APPL head in the DC, so there is no causative and possessive semantics.

Pylkkänen (2002) further develops the analysis of Marantz (1993), and argues that there are two APPL heads, which respectively constitute two different types of APPL constructions (Pylkkänen 2002: 19), one is High-APPL construction. The other is the Low-APPL construction. It is believed that the APPL construction in natural language can be divided into these two categories.

In Pylkkänen's analysis, the APPL head in the Low-APPL construction example (13b) indicates the transfer of ownership, and head connects its signifier DP (IO) and complement DP (DO), and its signifier DP can be the receiver (RECIPINT) or source (SOURCE) of the lower DP. The DOC of English is Low-APPL construction. In the High-APPL construction example (13a), the head of APPL indicates the relationship between the applicative argument (IO) and the event described by the verb. In this case, the VP is selected as the complement by the head of APPL; while in the Low-APPL construction, the DP is selected as the complement. In a language like English, the APPL head connects the applicative argument and the other argument (THEME), indicating the ownership relationship between the two arguments. There is no High-APPL construction in English. Pylkkänen believes that there is no APPL head in the DC, so that the DOC and the DC have an asymmetric structure, and the DOC has one more layer of APPL projection than the DC.

Anagnostopoulou (2003) also adopts the analysis of Marantz (1993) to study the DOC in Greek within the framework of APPL. Anagnostopoulou's research on the cliticization and double cliticization in Greek and other languages provides a theoretical basis for explaining the double cliticization in the DOC.

Miyagawa\&Tsujioka (2004), based on the research of Marantz (1993), use APPL construction to study the DOC in Japanese. They find that there are also two ditransitive constructions in Japanese through the analyses of quantifier domain and ambiguity in the DOC., and these two constructions have different argument structures (Miyagawa\&Tsujioka 2004: 6). There is no transformational relationship between them.

Soh (2005) uses the distributive quantifier 'each' as a test tool and applies the APPL framework to study the DOC in Mandarin Chinese, proving that the DOC has one more projection than the DC.

D. The G-parameter Approach

He (2008) proposes a parameterized functional category ' $G$ ' meaning 'transfer' to tackle the issue.

(14) [vP Subj v' [v [GP G' [G [VP IO V' [V DO]]]]]]

category ' $\mathrm{G}$ ' is postulate

The functional category ' $G$ ' is postulated to take on parameterized value as it might be null (as in English) or phonologically realized (as in Chinese, which can also be null). Parameterizing the functional category ' $G$ ' is helpful to reveal the universality and idiosyncrasy of the language and to explain the learnability of the language.

\section{THEORETICAL FRAMEWORK}


"A general trait of a minimalist approach to language is the endeavour to inspect and reduce any aspects (rules, filters, devices, formatives, etc.) that we regard as stipulative or unprincipled in the hope that such reduction will help us better understand the nature of this species-specific capacity (Gallego 2012: 10)." With this aim of the MP, Chomsky (2013, $2014,2015)$ publishes a series of papers to focus on the core syntactic properties of labels and further illustrate its applications in the generative grammar. Chomsky redefines the syntactic properties of labeling from two aspects of "the Third Factor Principle" (computational efficiency and interface conditions) and deeply interprets the theoretical explanatory power of the Labeling Theory and Labeling Algorithm (LA), systematically solving the fundamental theoretical problem of how to obtain the labeling for the merged categories, so that the Labeling Theory can follow the Strong Minimalist Thesis (SMT) ${ }^{1}$, which will inevitably have a profound impact on the development of generative grammar theory.

\section{A. The Simplest Merge}

According to the SMT, the only structure building operation of UG is Merge. Chomsky proposes the operation Merge: "One such operation is necessary on conceptual grounds alone: an operation that forms larger units out of those already constructed, call it Merge (Chomsky 1995: 296)." The labeling is nothing other than third-factor minimal search finding relevant object-identification information within the set that constitutes the output of (simplest) Merge (Epstein, Kitahara, \& Seely, 2017).

Merge is maintained in its simplest form in Chomsky $(2013,2014)$. The output of Merge is a syntactic object, and hence the output of Merge can also be one of the input arguments to Merge (as cited in Epstein et al. 2017). "For a syntactic object (SO) to be interpreted, some information is necessary about it: what kind of object is it? Labeling is the process of providing that information. Under PSG and its offshoots, labeling is part of the process of forming a syntactic object SO. But that is no longer true when the stipulations of these systems are eliminated in the simpler Merge-based conception of UG. We assume, then, that there is a fixed labeling algorithm LA that licenses SOs so that they can be interpreted at the interfaces, operating at the phase level along with other operations (Chomsky 2013: 43)."

Under SMT, therefore, the combinatorial operation of the generative procedure assumes (by hypothesis) the simplest formulation in what comes to be called "simplest Merge", a set-formation device that takes X and Y, and forms $\{\mathrm{X}, \mathrm{Y}\}$ (Epstein et al. 2017: 27).

(15) Merge $(X, Y)=\{X, Y\}$

Merge takes two (and only) two objects and puts them into the set $\{\mathrm{X}, \mathrm{Y}\}$, thereby creating the relation 'member of' for $\mathrm{X}$ and $\mathrm{Y}$ (as cited in Epstein et al. 2017). The operation Merge gives rise to hierarchical syntactic structure. Chomsky (2013) argues that Merge (X, Y) yields $\{$ X, Y $\}$ with no label projection or linear order. Collins (2017) also holds that it contains no information about linear order or syntactic category.

\section{B. Labeling Algorithm (LA)}

Chomsky $(2008,2013)$ proposes that Merge $(X, Y)=\{X, Y\}$, and that labels are identified via one of a small number of principles, the labeling algorithm.

For Chomsky $(2013,2014)$, labeling is the process of finding the relevant information within the set, $\{X, Y\}$, which identifies the categorial status of the entire set generated by simplest Merge. Labeling is "just minimal search, presumably appropriating a third factor principle, as in Agree and other operations" (Chomsky 2013) (as cited in Epstein et al. 2017). The Labeling is not stipulated in that it belongs to the third-factor principle. It is the name given to the result of an independently motivated minimal search procedure (Epstein et al. 2017: 29).

(16) Labeling Algorithm (Rizzi 2015: 321)

a. The category created by Merge receives the label of the closest head.

b. Labeling must be complete at the interfaces.

The LA includes two basic cases of Labeling: [H, XP] and [XP, YP].

(17) If $\mathrm{SO}=\{\mathrm{H}, \mathrm{XP}\}$ where $\mathrm{H}$ is a head and $\mathrm{XP}$ is not a head, then Label $(\mathrm{SO})=\mathrm{H}$.

(18) If $\mathrm{SO}=\{\mathrm{XP}, \mathrm{YP}\}$ and neither is a head, then

a. if XP is a lower copy, Label (SO) = Label (YP).

b. if Label (XP) and Label (YP) share a feature F by Agree, Label (SO) $=\langle\mathrm{F}, \mathrm{F}\rangle$. (Epstein et al. 2017)

Suppose first that the syntactic object (SO) is $\{\mathrm{H}, \mathrm{XP}\}, \mathrm{H}$ is a head and XP is not a head. Then $\mathrm{H}$ will be selected as the label by the minimal search to allow the object $\{\mathrm{H}, \mathrm{XP}\}$ to be identified as 'an $\mathrm{H}$ ' at the interfaces. As an example of (16), if Merge $($ see, $\{$ the, $\operatorname{man}\})=\{$ see, $\{$ the, $\operatorname{man}\}\}$, then Label $(\{$ see, $\{$ the, $\operatorname{man}\}\})=$ see. For Chomsky's labeling analysis, it follows naturally from 3rd factor minimal search and thus endocentricity relative to $\{\mathrm{H}, \mathrm{XP}\}$ is deduced, and without the postulation of an X-bar level of projection (Epstein et al. 2017).

Suppose then SO is $\{\mathrm{XP}, \mathrm{YP}\}$, neither a head. Here minimal search is ambiguous; search finds the sets XP, YP, neither of which is a head; it then searches further, finding both the head X of XP and the head Y of YP. It is assumed that this ambiguity is intolerable; left as is (an option available under free Simplest Merge), Full Interpretation (FI) is

\footnotetext{
1 The Strong Minimalist Thesis holds that "The optimal situation would be that UG reduces to the simplest computational principles which operate in accord with conditions of computational efficiency. This conjecture is sometimes called the Strong Minimalist Thesis (SMT) (Berwick\& Chomsky 2016: 94)."
} 
violated at the interface levels (Epstein et al. 2017). Chomsky (2013) puts forward a solution with two strategies: (A) modify SO so that there is only one visible head, and (B) X and Y are identical in a relevant respect, providing the same label, which can be taken as the label of the SO (Epstein et al. 2017: 31).

To summarize, Chomsky's (2013) analysis assumes that (Epstein et al. 2017: 31):

(i) Labels are required, but only at the interfaces.

(ii) Labeling is just minimal search.

(iii) There must be a single element that serves as the 'identifier' of a syntactic object, ambiguity of identification is not tolerated.

The output of Merge is label-free. Labels are determined by a labeling algorithm and play a role at the interfaces. Chomsky justifies the labeling algorithm by claiming that (as cited in Epstein et al. 2017): “...there is a fixed labeling algorithm LA that licenses SOs so that they can be interpreted at the interfaces... (Chomsky 2013: 43)."

\section{OUR ANALYSIS}

The Labeling Theory sheds new light on the analysis of the DOC. We will use the Labeling Theory to provide a tentative analysis for the DOC.

Take (1) a for example, repeated in (18).

(19) Zhangsan song Lisi yiben shu.

Zhangsan give Lisi one-CL book

'Zhangsan gave Lisi a book.'

A set is formed by using the "simplest Merge": Merge $(\mathrm{X}, \mathrm{Y})=\{\mathrm{X}, \mathrm{Y}\}$. Suppose 'song Lisi' 'give Lsi' functions as a complex verb, which is $\mathrm{X}$, with 'yiben shu' a book', which is $\mathrm{Y}$, as its object. The complex verb itself might be understood as the Pair-Merged SO 〈song, Lisi 〉, consisting of a verb and an adjunct.

(20) Merge(song Lisi, yiben shu) $=\{$ song Lisi, yiben shu $\}$

(21) Merge $(\mathrm{v}($ song Lisi, yiben $\mathrm{shu}))=\{\mathrm{v}$ \{song Lisi, yiben shu $\}\}$

Take $\mathrm{SO}_{\mathrm{i}}=\{\mathrm{v},\{\mathrm{V}, \mathrm{NP}\}\}$, viz., $\left\{\mathrm{v}\{\right.$ song Lisi, yiben shu $\}$. Here ' $\mathrm{v}$ ' as the label of $\mathrm{SO}_{\mathrm{i}}$ by minimal search in that $\mathrm{v}$ is unambiguously identifiable by applying (16); the head $\mathrm{H}$ in $\{\mathrm{H}, \mathrm{XP}\}$ is always found with "less search" than any feature-bearing element within XP. The pair-Merged element 'Lisi' is invisible for labeling in terms of Chomsky (2013, 2015) and the normal labeling procedure for [v [V, IA] $]$ will apply. As a result, $\{\mathrm{v}\{$ song Lisi, yiben shu $\}\}$ can be transferred and be interpreted at the interfaces of $\mathrm{CI}$.

Next, take $\mathrm{SO}_{\mathrm{j}}=\{\mathrm{NP},\{\mathrm{v}, \mathrm{VP}\}\}$, viz., $\{$ Zhangsan, $\{\mathrm{v},\{$ song Lisi, yiben shu $\}\}\}$. According to Epstein, Kitahara and Seely (2014), the minimal search here is ambiguous, locating two relevant heads, $\mathrm{N}$ and v. If the labeling of the SO fails and Full Interpretation is violated at CI in that CI cannot find the information it needs to identify the categorial status of this object; such identification of status is hypothesized by Chomsky (2013) to be a necessary prerequisite to CI (properly) interpreting the object. One way to label $\mathrm{SO}_{\mathrm{j}}$ is to raise NP to a higher position, which yields (after merger of $\mathrm{T}$ to $\mathrm{SO}_{\mathrm{j}}$ and subsequent subject raising) $\mathrm{SO}_{\mathrm{k}}=\{\mathrm{NP},\{\mathrm{T},\{\mathrm{NP}\{\mathrm{v}, \mathrm{VP}\}\}\}\}$ (Epstein et al. 2014). Chomsky (2013) takes the single NP (call it $\alpha$ ) in $\mathrm{SO}_{\mathrm{k}}$ to be in the domain $\mathrm{D}$ (in this case, in the set $\mathrm{SO}_{\mathrm{j}}$ now embedded within $\mathrm{SO}_{\mathrm{k}}$ ) if and only if every occurrence of $\alpha$ is a term of D. Given this, NP is taken to be not in $\mathrm{SO}_{\mathrm{j}}$ (a term of $\mathrm{SO}_{\mathrm{k}}$ ) because $\mathrm{SO}_{\mathrm{j}}$ does not contain every occurrence of NP as its term; rather, $\mathrm{NP}$ is taken to be in $\mathrm{SO}_{\mathrm{k}}$ because $\mathrm{SO}_{\mathrm{k}}$ contains every occurrence of $\mathrm{NP}$ as its term. Informally, the lower copy of NP is "invisible" when minimal search conducts a search for the label of $\{\mathrm{NP},\{\mathrm{v}, \mathrm{VP}\}\}$; it therefore "sees" only $\{\mathrm{v}, \mathrm{VP}\}$ when it "looks at" $\{\mathrm{NP},\{\mathrm{v}, \mathrm{VP}\}\}$. Thus, the movement of NP makes the $\mathrm{v}$ unambiguously identifiable; that is, minimal search finds the only "visible" head $\mathrm{v}$ as the label of $\mathrm{SO}_{\mathrm{j}}(\mathrm{Epstein}$ et al. 2014: 465-466).

$\mathrm{SO}_{\mathrm{k}}$, namely, $\{\mathrm{NP},\{\mathrm{T},\{\mathrm{NP}\{\mathrm{v}, \mathrm{VP}\}\}\}\}$, viz., $\{$ Zhangsan, $\{\mathrm{T},\{$ Zhangsan, $\{\mathrm{v},\{$ song Lisi, yiben shu $\}\}\}\}$, is of the form $\{\mathrm{XP}, \mathrm{YP}\} . \mathrm{SO}_{\mathrm{k}}$ is a set that contains two non-heads. Chomsky (2013) suggests that in such cases, the $\phi$-features shared by the two relevant heads $\mathrm{N}$ and $\mathrm{T}$ (in finite clauses) can be the label of $\mathrm{SO}_{\mathrm{k}}$; that is, when there are some prominent features shared by X and Y, minimal search can identify the label for $\{\mathrm{XP}, \mathrm{YP}\}$, namely, the features (in this case, the $\phi$-features) appearing on both heads $\mathrm{X}$ and $\mathrm{Y}$ (Epstein et al. 2014: 467). And then, $\mathrm{SO}_{\mathrm{k}}$ is transferred to CI for interpretation. The LA analysis of the DOC in Mandarin Chinese captures the direct-indirect asymmetry familiar from the traditional grammar.

\section{CONCLUSION}

This paper supplies a tentative study of the DOC in Mandarin Chinese. It justifies that the LA-the minimal search-provides an appropriate analysis of the DOC. The LA analysis of the DOC in Mandarin Chinese captures the direct-indirect asymmetry familiar from the traditional grammar.

\section{ACKNOWLEDGEMENTS}

This paper was funded by the International Training Program for Outstanding Young Scientific Research Talents in Colleges and Universities of Department of Education of Guangdong Province. It is supported by 2019 Project of National Social Science Fund of China "Cognitive Motivation and Inter-language Processing Mechanisms of 
Non-argument Structures in English and Chinese" (19BYY095).

\section{REFERENCES}

[1] Anagnostopoulou, E. (2003). The Syntax of Ditransitives: Evidence from Clitics. Berlin: Moutonde Gruyter.

[2] Aoun, J.\&Y-H. A. Li. (1989). Scope and Constituency. Linguistic Inquiry 20:141-172.

[3] Baker, M. (1988). Incorporation: A Theory of Grammatical Function Changing. Chicago: University of Chicago Press.

[4] Barss, A.\&H. Lasnik. (1986). A note on Anaphora and Double Objects. Linguistic Inquiry 17: 347-354.

[5] Beck, S.\&K.Johnson. (2004). Double Object Again. Linguistic Inquiry 35: 97-124.

[6] Berwick, R. C., \& Chomsky, N. (2016). Why Only Us: Language and Evolution. MIT press.

[7] Bowers, J. (2011). Non-event nominals and argument structure. Lingua, 121(7), 1194-1206.

[8] Chomsky, N. (1975). Reflections on Language. New York: Pantheon.

[9] Chomsky, N. (1995). The Minimalist Program. Cambridge, MA: MIT Press.

[10] Chomsky, N. (2008). On phases. In Freidin, R., Otero, C.P., \& Zubizarreta, M.L. (Eds.). Foundational Issues in Linguistic Theory: Essays in Honor of Jean-Roger Vergnaud. MIT, Cambridge, MA: 133-166.

[11] Chomsky, N. (2013). Problems of Projection. Lingua (130): 33-49.

[12] Chomsky, N. (2014). Problems of projection: extensions. In Di Domenico E. et al. (eds.). Structures, Strategies and Beyond. Amsterdam/ Philadelphia: Benjamins Publishing Company: 1-16.

[13] Chomsky, N. (2015). Some core contested concepts. Journal of Psycholinguistic Research (44): 91-104.

[14] Collins, C. (2017). Merge (X, Y) $=\{X, Y\}$. In Bauke, L., \& Blümel, A. (Eds.). Labels and Roots, 128, 47-68.

[15] Epstein, S. D., Kitahara, H., \& Seely, T. D. (2014). Labeling by minimal search: Implications for successive-cyclic A-movement and the conception of the postulate "phase". Linguistic Inquiry, 45(3), 463-481.

[16] Epstein, S. D., Kitahara, H., Seely, T. D. (2017). Merge, labeling and their interactions. In Bauke, L., \& Blümel, A. (Eds.). Labels and Roots, 128, 17-45.

[17] Gallego, Á. J. (2012). Introduction: A framework of phases for linguistic theory. In Gallego, Á. J. (Ed.). Phases: Developing the framework, 9-44. De Gruyter Mouton.

[18] Green, G.M. (1974). Semantics and Syntactic Regularity. Bloomington/London: Indiana University Press.

[19] Harley, H. (2003). Possession and the double object construction. In Pica, P., \& Rooryck, J. (Eds.). Yearbook of Linguistic Variation, vol. 2. Amsterdam: Benjamins: 31-70.

[20] He, Xiaowei. (2003). The Relationship between the Double Object Construction and the Dative Construction. Journal of Foreign Languages (2): 25-31.

[21] He, Xiaowei. (2008). A minimalist approach to the generation of the double object construction in English and Chinese. Modern Foreign Languages (1): 1-12.

[22] He, Xiaowei. (2009). A semantic study of the ditransitive construction. Foreign Language Teaching and Research (1): 18-24.

[23] Jackendoff, R. (1990). On Larson's Treatment of the Double Object Construction. Linguistic Inquiry 21:427-456.

[24] Kayne, R. (1984). Connectedness and Binary Branching. Dordrecht: Foris.

[25] Larson, R. (1988). On the Double Object Construction. Linguistic Inquiry 19: 335-391.

[26] Larson, R. K. (1990). Double objects revisited: Reply to Jackendoff. Linguistic inquiry, 21, 589-632.

[27] Marantz, A. (1993). Implications of Asymmetries in Double Object Construction. In Mchombo, S. (ed.). The Theoretical Aspect of Bantu Grammar. Stanford: CSLI Publications: 113-150.

[28] Miyagawa, S.\&T. Tsujioka. (2004). Argument Structure and Ditransitive Verbs in Japanese. Journal of East Asian Linguistics 13: $1-38$.

[29] Pesetsky, D. (1995). Zero Syntax: Experiencers and Cascades. Cambridge, MA: The MIT Press.

[30] Pylkkänen, L. (2002). Introducing Argument. Ph.D. diss., MIT.

[31] Rizzi, L. (2015). Cartography, criteria, and labeling. In Shlonsky, U. (ed.). Beyond Functional Sequence: The Cartography of Syntactic Structures. University of Siena and Geneva.

[32] Soh, H. L. (2005). Mandarin Distributive Quantifier GE 'each': The Structures of Double Complement Constructions and the Verb-preposition Distinction. Journal of East Asian Linguistics 14:155-173.

Haojie Li was born in Shandong, China in 1974. He received his M.A. degree in Sichuan International Studies University in 2005 and he is a $\mathrm{PhD}$ candidate majored in English Language and Literature at Guangdong University of Foreign Studies.

$\mathrm{He}$ is currently a lecturer in the School of Foreign Languages, Southwest University of Political Science and Law, Chongqing, China. His research interests include syntax and theoretical linguistics. He has published a number of studies in his areas of expertise, which have appeared in scholarly publications in Mainland China and Europe.

Zhigang Ma was born in Gansu, China in 1971. He received his M. A. degree and PhD degree in Guangdong University of Foreign Studies, China. He is currently an editor of the Journal Modern Foreign Languages and Professor of Linguistics at the Center of Linguistics and Applied Linguistics in Guangdong University of Foreign Studies. His research interests include syntax and second language acquisition. He has published numerous papers in international journals and journals in China. He is the corresponding author of this study, which is supported by 2019 Project of National Social Science Fund of China "Cognitive Motivation and Inter-language Processing Mechanisms of Non-argument Structures in English and Chinese” (19BYY095). 